\title{
然
}

Antonio M. Ávila Álvarez*

Miguel Ángel Díaz Mier**

\section{LA EVOLUCIÓN DE LA OMC EN LA DÉCADA DE 2010}

En este trabajo se pretende obtener una visión del desarrollo de la OMC en el decenio de 2010. Se parte de un acontecimiento que se considera clave como es la celebración de la cuarta Conferencia Ministerial de Doha y los compromisos acordados en la misma. La actualización en muchas de sus rúbricas resulta más que notable, si bien no se ha logrado la puesta en práctica de otras. Se resaltan las nuevas características de la institución, cuyo conocimiento resulta hoy necesario en todo análisis de la economía mundial.

Palabras clave: Conferencias Ministeriales, OMC, institución, cooperación.

Clasificación JEL: F10, F13, F19.

\section{Introducción}

En 2008 tuvimos ocasión de coordinar y publicar en las páginas de la revista mensual de Información Comercial Española (en el número 843 de julio-agosto) un volumen dedicado, en su integridad, a la consideración de los principales conceptos que habían configurado la estructura e historia del que se había denominado sistema GATT-OMC. En dicho número participaron destacados especialistas sobre los temas que integraba tal sistema, todos ellos vinculados a universidades españolas, así como a la operativa práctica en la institución creada tras la conclusión de la Ronda de Uruguay (1986-1994).

\footnotetext{
* Doctor en Derecho y Economía.

** Técnico Comercial y Economista del Estado.

Versión de octubre de 2017.
}

Desde entonces ha transcurrido un número significativo de años y acontecimientos, lo que, en nuestra opinión, permite realizar un análisis a modo de resumen de la que ha sido la evolución de la OMC en el período al que se refiere el título de este trabajo, esto es, la década de 2010. En el mismo pretendemos realizar un examen del cumplimiento de los objetivos señalados en las Conferencias Ministeriales que han tenido lugar en ese período, pero especialmente nos fijamos en dos Conferencias anteriores al mismo tiempo (la de Doha y la de Hong Kong). También buscamos realizar una exposición mínima de las cuestiones que de modo singular ha desarrollado la literatura técnica recogida en el número indicado del ICE y actualizada a lo largo de la década señalada.

Para realizar ambos objetivos partimos de la enumeración de los temas que se relacionan en el anexo a este trabajo y que resulta $\triangleright$ 
sencillo indicar, los incluidos en la Conferencia Ministerial de Doha del año 2001 (siglas WT/ MIN/01/DEC) y actualizados en la sexta Conferencia de 2005 (celebrada en Hong Kong y que lleva las siglas WT/MIN/05/DEC). La primera de estas despertó la floración de trabajos de todo tipo y posiciones, tanto en el mundo de los negociadores comerciales internacionales como en los ámbitos académicos y en general en los públicos, seguidores de los acontecimientos de la vida internacional. Buena parte de ello se debía al objetivo de desarrollo económico que bautizó, bajo esa denominación, a la Conferencia de Doha al lanzar sus objetivos. Recordemos que se trataba de iniciar, por primera vez dentro de la OMC, una Ronda de Negociaciones (habían sido ocho las que el GATT había amparado).

Además, la Conferencia de Doha buscaba, con el iniciado lanzamiento de la Ronda, el logro de un éxito que hiciera olvidar el fracaso de la Conferencia de Seattle, en la que se habían manifestado posiciones anti OMC, especialmente vinculadas a los movimientos antiglobalización. Señalamos que también en la época se hablaba del «milenio del desarrollo», que ocuparía un destacado lugar dentro de las actividades de las principales instituciones económicas internacionales.

De modo inicial nos interesa realizar una sencilla comparación cuantitativa para mostrar que entre 2001 y 2005 se habrían producido mejoras en la simple presentación de los temas. Así, en el citado documento de la Conferencia de Doha, en sus 11 páginas, se incluían 52 párrafos de definición de temas, mientras que pocos años después (en 2005) se encuentran en 57 páginas, 59 párrafos, con estructura similar a las de Doha. Quiere ello decir que en la Conferencia de Hong Kong se lograba una mayor precisión en la primera enumeración y, además, se incluían dentro de la Ronda nuevos temas. En el anexo los hemos indicado con una llamada.

Evidentemente, una de las formas de proceder para nuestro estudio hubiera sido realizar un análisis temporal, poniendo de relieve lo ocurrido en las Conferencias Ministeriales que se han celebrado desde 2005. Ahora bien, recordemos que, según lo previsto en el Tratado de Marraquech por el que se creaba la OMC, junto a la definición de las funciones de las Conferencias en el ámbito institucional, se señalaba que las mismas debían celebrarse ordinariamente cada dos años. Aquí señalamos que, en sentido estricto, esto no se ha cumplido. Para mostrarlo, apuntamos que entre las Conferencias de 2007 y 2011 (celebradas en Ginebra) debía haber tenido lugar otra antes de las que se celebraron en Bali (2013) y Nairobi (2015).

En la actualidad está en plena preparación la Conferencia de Buenos Aires, que se celebrará en diciembre de 2017. Con ello, se recoge una de las tradiciones no escritas de la OMC de celebrar cada Conferencia en lugares de continentes distintos.

Un primer estudio de las actas de las Conferencias, celebradas entre 2005 y 2015 , pone de relieve que en todas ellas se realizan declaraciones sobre el fortalecimiento del sistema multilateral del comercio y su mantenimiento. Sin embargo, los exámenes sobre el cumplimiento de los objetivos de la Ronda de Doha ponen de manifiesto que, con excepción del Acuerdo sobre Facilitación del Comercio, conseguido en 2015, se ha avanzado en pocos temas.

De ahí que, una vez que no hemos adoptado el procedimiento indicado, hayamos optado por una presentación más tradicional. Dividiremos así el trabajo, después de la introducción, en dos partes. En la primera de ellas haremos una presentación de temas de carácter general $\triangleright$ 
para dedicar la segunda al estudio de la situación de algunos temas indicados en el anexo. Destacaremos, en la medida de lo posible, con ayuda de las informaciones que proporciona la $\mathrm{OMC}$, algunos datos cuantitativos para ilustrar los temas.

\section{Aspectos generales}

Bajo esta rúbrica incluimos una serie de cuestiones que nos parece han tenido en el período de estudio un papel que merece destacarse, bien porque han experimentado algún tipo de mejora respecto a lo estudiado en el trabajo publicado en 2008 , bien porque han sido destacadas en las obras de los especialistas en temas de OMC, aunque, como puede deducirse de nuestras primeras observaciones, aún no se haya llegado a algún tipo de acuerdo expresado en la forma tradicional del sistema heredado del GATT, esto es, en los instrumentos jurídicos con los que se cierran las rondas negociadoras.

1. Para nosotros, sin ninguna duda, la principal mejora que se ha presentado en la OMC es la de la transparencia en sus actividades. Así, queremos destacar que, después de una serie de experiencias, hoy podemos contar con una página web de empleo relativamente sencilla. En ella se puede encontrar informaciones sobre cuestiones de carácter general (por ejemplo, la situación de los miembros de la OMC, que hoy suman 164 , entre los que se incluyen las principales potencias comerciales del mundo). A su lado se hallan noticias sobre las diferentes actividades que se llevan a cabo en la institución, las cuales se presentan en los tres idiomas oficiales de trabajo. En las informaciones destacamos, de modo especial, las rúbricas sobre cuestiones comerciales en sentido amplio, pues no solamente se refieren a intercambio de mercancías, sino que incluyen el comercio de los principales tipos de servicios y los aspectos comerciales de los derechos de propiedad intelectual. Igualmente, son significativas las informaciones estadísticas y, sobre todo, los documentos jurídicos en que se expresan los trabajos de la institución, especialmente las informaciones actualizadas sobre los distintos Acuerdos de la Ronda de Uruguay.

Han de destacarse, también, las completas documentaciones de los casos planteados dentro del sistema de solución de diferencias a las que nos referimos en la siguiente parte de este trabajo. Todo ello contrasta con las dificultades que hasta hace relativamente poco tiempo existían para conocer las actividades de la OMC. En la práctica, con excepción lógica de las informaciones que son confidenciales hoy (las que, por ejemplo, recogen posiciones de negociación que así lo requieren), procede hablar de la posibilidad de conocer cierto acontecer en la OMC, y no son excepciones las Conferencias Ministeriales y el desarrollo de las negociaciones multilaterales.

2. También en nuestra opinión debe destacarse la muy notable mejora de las relaciones de la OMC con las principales instituciones económicas internacionales (Fondo Monetario Internacional, Banco Mundial, UNCTAD, pero también OCDE y Unión Europea). Ello se manifiesta de modo especial en la realización de proyectos conjuntos. 
En este orden subrayamos los trabajos realizados en la esfera de los servicios y su comercio. Así, cabe señalar la realización de un manual necesario sobre estadísticas de servicios en el que participaron FMI, UNCTAD, Comisión Europea y OCDE. Y en este orden se encuentran las completas estadísticas (I-TIP).

Con la OCDE se desarrolla una actividad incluida en la rúbrica «Ayuda para el Comercio".

De forma especial, dentro del concepto de «Vigilancia del Comercio», la OMC, conjuntamente con OCDE y UNCTAD, realiza dos informes semestrales en los que se ponen de manifiesto las medidas proteccionistas adoptadas o concluidas y su vigencia por parte de los países que constituyen el llamado Grupo de los 20 (G20), en el que están incluidos los principales países que intervienen en el comercio mundial. Tales informes conjuntos se realizan desde la última crisis mundial y demuestran los deseos de actuar en unión para solucionar problemas y prever respuestas a lo que pudiera plantearse más adelante.

Las relaciones con el Fondo Monetario Internacional, de Naciones Unidas (debe recordarse que la OMC no forma parte del sistema institucional de las mismas), permiten hoy a la OMC confeccionar información de primer orden en el dominio estadístico. También dentro de los Comités que administran los Acuerdos de la Ronda de Uruguay (especialmente Derechos de Propiedad Intelectual, Aranceles, Servicios Aéreos), la OMC coopera con las Instituciones de Naciones Unidas que tienen las principales competencias en las órdenes señaladas.
3. Otro aspecto que se discute sobre la OMC ya desde su creación, que ha sido abordado por diferentes especialistas tanto en el orden académico como el operativo (recordemos la cooperación de P. Sutherland en el texto coordinado por Macrory y otros en su disección del trabajo sobre el futuro de la OMC), es la mejora del sistema institucional. Ahora bien, en nuestra opinión, pese a las discusiones sobre estos temas, no han sufrido modificaciones. El principal de ellos es el relativo al sistema de toma de decisiones en el que sigue vigente el método heredado del GATT como es el consenso. Otros temas relacionados con la participación de los países en las elaboraciones de las discusiones siguen presentando las dificultades que experimentan los países que no cuentan con instrumentos de presencia en Ginebra para estar en los órganos de discusión. Finalmente, las diferentes propuestas para establecer órganos de consulta para la dirección de la OMC no han sido llevados a la práctica.

4. Nos han sorprendido algunos aspectos de la literatura científica o académica de los estudios de la OMC. De hecho, hemos de destacar que los grandes manuales omnicomprensivos de todo el conjunto de los temas (por ejemplo, las obras de Koekman y Kostecki, de Matsushita et al. o de Trebilcock) han publicado, en este decenio, ediciones actualizadas que parten de un examen en profundidad de la Ronda de Doha y de una introducción de los llamados «nuevos temas", como comercio y medio ambiente; comercio y derechos humanos. A su lado, sin embargo, encontramos obras monográficas sobre algunos $\triangleright$ 
temas y también aplicaciones sobre el significado de la OMC en distintos ámbitos de estudio sobre comportamiento empresarial y académico. En este último orden, en los manuales sobre economía mundial utilizados en las universidades españolas, pueden encontrarse descripciones de la OMC con niveles similares a los de otras instituciones como el FMI, el Banco Mundial, etcétera. Especialmente significativas son las que hacen referencia a la Conferencia de Bretton Woods y al nacimiento del GATT.

Hemos de destacar que en los estudios de orden empresarial que se han publicado en la última década, en diferentes campos, se encuentran tratamientos más que aceptables de la OMC. Así, en obras sobre marketing internacional, negocios internacionales o sobre el entorno internacional se pueden hallar tales tratamientos que, lógicamente, en otro orden de trabajos no faltan en obras sobre globalización e, indudablemente, en las destinadas a la economía política internacional. Algunas de las mismas se han incluido en la bibliografía.

5. Una cuestión que ha preocupado siempre a los expertos en el sistema GATTOMC desde el nacimiento del mismo, es el tratamiento del regionalismo comercial, entendido en el sentido de los diversos acuerdos existentes en el ámbito de las relaciones comerciales mundiales que se refieren a un número reducido de países y que revisten diferentes formas, aunque las más frecuentes sean los acuerdos de libre comercio y las uniones aduaneras.

El sistema ha intentado buscar fórmulas de compromiso, especialmente el sometimiento a los métodos que ha desarrollado el artículo xxIv del GATT y que hoy se encuentran dentro de procedimientos de modificación. Pues bien, la OMC informa que en la actualidad han sido notificados 445 acuerdos comerciales regionales de diversos tipos. Nosotros hemos observado que en la década que examinamos no se han producido de modo significativo nuevos acuerdos.

Sin duda, las discusiones sobre la compatibilidad del sistema multilateral de comercio con estos acuerdos siguen siendo materia de análisis, pero no hemos encontrado datos estadísticos significativos sobre cuánto aumentan 0 disminuyen los acuerdos regionales las cifras de comercio sometidas a las reglas multilaterales.

6. Desde una perspectiva fundamentalmente académica, uno de los temas que es de actualidad permanente en el ámbito de la OMC, si bien de difícil solución operativa, se refiere a los temas relativos a la soberanía nacional. En nuestro caso ello ha venido motivado por el hecho de que algunas de las resoluciones adoptadas en el proceso de solución de diferencias de la OMC han provocado reacciones por parte de grupos que consideraban que las mismas erosionaban normas nacionales.

En este orden, destacados especialistas, como los profesores Jackson y Petersman, se han referido a esta cuestión examinando diversos aspectos como la evolución del concepto de nación-Estado, los ámbitos en que se manifiesta la soberanía, las relaciones de cooperación internacional y su impacto sobre $\triangleright$ 
la misma, y la subsidiariedad entre otros aspectos. Se ha señalado que «la OMC tiene competencia y facultades que antes eran de monopolio de los Estados» y que lo importante es «el balance entre las pérdidas de espacio normativo a nivel nacional y las ventajas de la cooperación", y si el imperio de la ley a nivel multilateral es positivo o negativo.

7. Una cuestión de permanente actualidad en la OMC es el tratamiento de los países en desarrollo, entre los que hoy se distinguen distintos grados. Lo básico es la forma de hacer operativa la definición de «trato especial y diferenciado». Así, la Ronda de negociaciones lanzada en la Conferencia de Doha ha supuesto que en todas las Conferencias posteriores se hayan examinado las formas de hacerlo efectivo.

En esta línea, por una parte se encuentran las formas de tratamiento a estos países en los acuerdos establecidos en la Ronda de Uruguay, cuya formulación jurídica, en forma de ampliación de plazos o similares para la plena aceptación, resulta fácil de sintetizar (véase WT/ COM TD/W 196).

Pero, en nuestra opinión, lo que resulta destacable en la década que examinamos se refiere a la "ayuda para el comercio», que, como señalamos anteriormente, es una iniciativa conjunta de OMC y OCDE. Todo ello se encuentra bajo el enunciado «creación de capacidad comercial», que comprende, además, otras actividades.

8. Nos parece enormemente significativo en esta enunciación de actividades de la OMC en la década de 2010, y como complemento de cuanto hemos señalado, a la vez que como una demostración de la evolución desde el primer GATT, el fortalecimiento del rigor jurídico de las normas de la OMC, expresadas inicialmente en la formulación de los acuerdos, pero de modo especial en las fórmulas de desarrollo de las actividades y en el cumplimiento de las obligaciones que se manifiestan en el sistema de solución de diferencias al que nos referiremos posteriormente.

\section{Algunos temas específicos}

Nos referimos aquí a determinados temas incluidos en la relación del anexo. No podemos estudiar todos ellos, pero queremos destacar los que en la década han supuesto un mayor avance para el cumplimiento de los objetivos ya indicados en el programa de negociaciones comerciales de Doha.

1. Así, nos referimos, en primer lugar, a la facilitación del comercio porque, como dijimos, entre los temas que se expresan en el anexo, es el único que ha fructificado en forma de un acuerdo que, tras haber sido sometido a los procesos de ratificación de los países miembros, ha sido aprobado por 122 , superando la cifra necesaria de 100.

Examinamos en esta cuestión, en primer término, los objetivos de las negociaciones que se iniciaron en agosto de 2004 dentro del programa de Doha. Para ello se pretendía aumentar la transparencia de la normativa que viene expresada desde 1948 en el artículo c del GATT; simplificar los procedimientos de importación, exportación y los de aduanas; $D$ 
lograr una aplicación efectiva de la libertad de tránsito que se define en el artículo v del GATT; conseguir una auténtica cooperación entre autoridades aduaneras o las competentes en esta materia; $y$, finalmente, responder en este orden al trato especial y diferenciado para los países en desarrollo.

La importancia de las cuestiones se derivan de la simple exposición de las materias que se encuentran en las operaciones prácticas que recogen, además de las aduaneras, el control de las medidas sanitarias y fitosanitarias relativas a importación y exportación, el transporte, la gestión de puertos y servicios de seguridad. Todo ello se plasma, además, en las documentaciones necesarias, lo que se refleja en una etapa de desarrollo de las operaciones comerciales asociadas a la globalización acrecentada, no solo en los intercambios de mercancías, sino también en los de informaciones.

De una forma simplificada, el Acuerdo de Facilitación del Comercio (AFC) contiene disposiciones para agilizar el movimiento, el levante y el despacho de carga, incluidas las mercancías en tránsito. Se divide en tres secciones: en la primera se encuentran los criterios para agilizar los despachos aduaneros y el flujo internacional de mercancías, así como la cooperación aduanera; en la segunda se tratan los países en desarrollo, recogiendo la distinción más creciente entre «países en desarrollo y países menos desarrollados» y señalando, para ellos, el tratamiento más favorable y la asistencia técnica; en la tercera, que no se aparta de los sistemas operativos de posicionamiento de los Acuerdos de la Ronda de Uruguay, se establece el mecanismo institucional (creación de un Comité de Facilitación del Comercio), si bien como novedad se pide a los países miembros la creación de comités nacionales.

Resulta de interés indicar las funciones de dichos comités. Estas son las destinadas a negociar, coordinar y fomentar la facilitación del comercio por medio de la simplificación, coordinación, estandarización o armonización de los procedimientos comerciales y aduaneros. Téngase en cuenta que se estima que en una transacción aduanera media intervienen, según el Banco Mundial, del orden de 20 a 30 actores diferentes, 40 documentos y 200 elementos de datos (muchos de los cuales se repiten). Pues bien, los comités tienen ante sí tareas importantes de coordinación de los sectores público y privado, los diversos ministerios de las Administraciones, las autoridades aduaneras, los agentes de aduanas junto con los comerciantes, los industriales, los transitorios y los operadores de transporte del mundo privado. A efectos prácticos, conviene indicar que la Unión Europea ratificó, en 2015, el acuerdo, y que lógicamente en el Código Aduanero, establecido en 2016, se llevan a la realidad los objetivos de facilitación. Por otro lado, en cumplimiento de los aspectos de ayuda al desarrollo, especialmente en lo que se refiere a asistencia técnica, se ha prometido dotar a la misma, para el período de cinco años, con 400 millones de euros. Un análisis sobre el acuerdo examinado, a realizar en la Conferencia de Buenos Aires, será elemento importante para efectuar una valoración adecuada. 
2. Un segundo tema, que para nosotros tiene una especial significación y al que nos hemos referido en la anterior parte de este trabajo, es el sistema de solución de diferencias. Partimos de un estudio anterior realizado por Miguel Ángel Díaz Mier en el que se presenta la evolución de tal sistema desde las primeras actividades del GATT para llegar, en el Acta de Marraquech (1994), al que se denominó «Entendimiento relativo a las normas y procedimientos por los que se rige la solución de diferencias». Es relativamente sencillo conseguir un esquema que lo explica, en la página web de la OMC, que, por otra parte, señala que «es la piedra angular del sistema multilateral de comercio y una contribución excepcional de la OMC a la estabilidad de la economía mundial».

En efecto, el procedimiento que recoge en buena parte la búsqueda de formas de solventar las diferencias entre los Estados, en relación con sus prácticas de política comercial en sentido amplio, fortalece el sometimiento a normas (aspectos que hemos subrayado muchas veces), porque es la clave de todo el aparato institucional que ha de aplicarse. Nos interesa señalar, en primer lugar, mediante una serie de datos obtenidos de la compleja relación de casos presentados, que sin duda requieren un examen a fondo, el llegar a conocer lo que adecuadamente se ha denominado jurisprudencia de la OMC.

Así, sin entrar en la situación de cada uno de los procedimientos, cabe decir que, a principios de septiembre de 2017, se han presentado 530 casos. Estos se refirieron tanto a mercancías (231) como a propiedad intelectual (13) y a servicios (28). A título de ejemplo, entre los casos de mercancías, figuran los relativos a regímenes fiscales aplicables a importaciones y a exportaciones, las prescripciones en materia de pruebas y certificación, los problemas con patentes y productos como el pentapropileno (caso muy complejo de diferencias entre Estados Unidos y la Unión Europea), y los problemas en cuanto a propiedad intelectual, que se refieren a los conceptos que se incluyen en el ADPIC como las patentes y las indicaciones geográficas. En servicios, cabe señalar diferencias en materia de distribución, enseñanza e ingeniería. Todo ello ha dado origen a numerosas publicaciones. Además, pueden encontrarse las relaciones de países miembros que han actuado como reclamantes o reclamados. No debe extrañar que sean Estados Unidos y la Unión Europea los que tienen mayor número de asuntos, aunque cada vez hay mayor número de países en desarrollo que acuden al procedimiento $y$, curiosamente, lo hacen también países que forman parte de un acuerdo regional para solucionar temas que el acuerdo de este tipo no logra resolver y que revela la fortaleza del sistema de solución de diferencias.

En esta breve síntesis de los temas que forman el conjunto de actividades de la OMC, los aspectos del sistema de la solución de diferencias ocupan un lugar especial, aunque son discusiones de carácter muy técnico, y de hecho en 2017 solo podemos hablar de una serie de aspectos específicos contenidos en un documento de 2016. Ahora bien, sí merece la pena recordar los orígenes de $D$ 
esta situación: los miembros de la OMC no llegaron hasta 2001 (Conferencia de Doha) a proseguir el examen sobre mejoras y aclaraciones del entendimiento señalado ya en el Acta de Marraquech. De hecho, en 2017, solo encontramos documentación sobre reuniones informales que han permitido estructurar las discusiones en doce cuestiones temáticas que se refieren a aspectos de posibles mejoras de Entendimiento. Encontramos, entre ellas, los derechos de terceros, los plazos y el cumplimiento efectivo.

3. Entre los temas que se incluyen en la relación que se recoge en el anexo de este trabajo figuran por separado «agricultura» $y$ «acceso a los mercados para productos no agrícolas», conocido por las siglas AMNA. Nos permitimos presentarlos en un mismo epígrafe porque su tratamiento en la Ronda de Doha ha seguido caminos paralelos, si bien parece claro que, tanto por las cantidades de documentos generados como por las discusiones públicas, los temas de agricultura tienen un mayor espacio.

Refiriéndonos en primer lugar al AMNA, hemos de destacar que, en la teoría, las negociaciones se centran en los aranceles. Ahora bien, en la práctica los responsables de las negociaciones AMNA han seguido el desarrollo de las negociaciones sobre agricultura al que nos referiremos a continuación.

Debe indicarse que el Acuerdo sobre Agricultura pactado en las negociaciones de la Ronda de Uruguay exigía lograr a largo plazo reducciones sustanciales y progresivas de la ayuda y de la protección otorgada en la esfera de la agricultura, y que a ese respecto las negociaciones debían reanudarse en el año 2000. Sin embargo, en la práctica, solo se consiguió un paso adelante con el lanzamiento de la Ronda de Doha, cuyo desarrollo, como hemos indicado, forma parte de los trabajos de los principales tratadistas de la OMC. Pero también, de hecho, solamente en 2004 se acordó un conjunto de decisiones (el llamado «paquete de julio») en el que se contenía, sobre agricultura, un «marco» que experimentaría incisivas transformaciones sobre un texto que contenía propuestas para la reducción de aranceles para productos agrícolas y de las subvenciones en materia de tales productos. Se realizaron en 2007 y 2008 revisiones sobre el mismo, llegándose, en diciembre de 2008, a un documento de «proyecto revisado de modalidades para la agricultura», muy amplio (140 páginas), adoptado en una sesión extraordinaria del Comité de Agricultura (siglas TN/AG/W/4/REV5.4). Son muchos los temas que han ocupado las actividades del señalado Comité de Agricultura. De un modo resumido se citan los siguientes:

i) Aranceles, contingentes y salvaguarda, dentro de los cuales destacamos el cálculo de los equivalentes ad valorem, los métodos de administración y las tasas de utilización de los contingentes arancelarios.

ii) Ayuda interna asociada a los criterios establecidos en el Acuerdo sobre subvenciones (los llamados compartimentos distinguidos por su color verde, azules y ámbar): se han estudiado las ayudas de minimis y las medidas globales de la ayuda para productos específicos y la total. 
iii) Subvenciones a la exportación, incluyendo los créditos a la exportación.

iv) Las situaciones de los países en desarrollo.

Han de destacarse, en el examen de lo acontecido en la década de 2010, dos Conferencias Ministeriales en el dominio de la agricultura, la de Bali (2013) y la de Nairobi (2015), que comentamos.

En la primera de ellas se incluyeron varias e importantes decisiones:

a) Acuerdo para mejorar una solución respecto a la constitución de existencias públicas en fines de seguridad alimentaria y de abstenerse de poner en tela de juicio las infracciones que se produzcan como resultado de los programas de constitución de existencias públicas con fines de seguridad alimentaria de los países en desarrollo.

b) Mayor transparencia en la administración de contingentes arancelarios, especialmente que los Gobiernos no creen obstáculos al comercio por la forma de distribución de tales contingentes.

c) La ampliación de los denominados «servicios generales", incluyendo entre ellos los gastos correspondientes al uso de las tierras, la reforma agraria, la gestión de los recursos hídricos y otros programas de reducción de la pobreza.

v) Una declaración destinada a la reducción de todas las formas de subvenciones a la exportación.

Desde luego, la Conferencia de Nairobi ha conseguido el logro de una decisión tan importante como ha sido la eliminación total de las subvenciones a la exportación de productos agropecuarios y el establecimiento de disciplinas sobre las medidas relativas a la exportación que tengan efectos equivalentes. Han destacado los informes de la OMC que «la eliminación indicada de las subvenciones» ha alcanzado una meta fundamental del objetivo de desarrollo sostenible «como es el hambre cero». Y, por otra parte, en la Conferencia se avanzaría en el tema ya recogido en Bali como es la utilización, por parte de países en desarrollo, de los programas de constitución de existencias púbicas con fines de seguridad alimentaria.

En definitiva -y según nuestro criteriola seguridad alimentaria se constituye como un tema de especial cumplimiento por el tratamiento desfavorecido para los países en desarrollo.

4. Nos ha preocupado, de un modo especial, el tratamiento en la OMC de los derechos de propiedad intelectual relacionados con el comercio, como lo muestra el trabajo que publicamos en febrero de 2017 en este boletín de Información Comercial Española. De igual forma, ha de señalarse que las tareas que se llevan a cabo en el ADPIC han proseguido en la década en sus niveles técnicos y especialmente positivos en la cooperación con la Organización Mundial de la Propiedad Intelectual. Pero no encontramos soluciones espectaculares en materias como las indicaciones geográficas, las transformaciones de tecnología o el cambio climático, además de cuestiones relacionadas con la protección de plantas y animales, los conocimientos tradicionales y la biodiversidad. 
El trabajo señalado mostraba algunas de las cuestiones indicadas que se ha plasmado en estudios monográficos como el realizado conjuntamente por las Organizaciones Mundiales de Salud y de Propiedad Intelectual junto a la OMC, sobre acceso a tecnología e innovación en medicina y a las intersecciones entre salud pública, propiedad intelectual y comercio.

5. Por último, los trabajos sobre los distintos acuerdos negociados en la Ronda de Uruguay que se expresan en el anexo, así como otras nuevas áreas de negociación como la financiación del comercio, el comercio electrónico y, de modo especial, los temas relativos a medio ambiente que son, en la década de 2010, sencillamente objeto de mención.

\section{Conclusión}

En una economía global, que ha sufrido una grave crisis económica y financiera, con intensidad desigual por áreas geográficas, y a diferencia de lo que ocurrió en 1929 con todas las dificultades y problemas agravados por decisiones políticas en los últimos años, la OMC ha sido, y esperamos que sea, un instrumento, con todas sus eficiencias, básico para mantener un sistema comercial abierto y multilateral en el que, a veces, con desesperante lentitud, se siguen dando pasos que fortalecen y facilitan el intercambio, como el Acuerdo de Facilitación de Comercio en el que se nos antoja básico el buen funcionamiento del sistema de solución de diferencias que, en definitiva, supone la aplicación del derecho internacional sobre los intereses nacionales.

\section{Bibliografía}

[1] ALBAUM, G.; DUERR, E. y JONASSEN A. (2016). International Marketing and Export Management (8. $\cdot^{\mathrm{a}}$ ed.). Pearson.

[2] ÁVILA ÁlVAREZ, A.M. (2008). «La participación del sistema GATT-OMC en la gobernanza mundial». Revista ICE, n. .843 .

[3] ÁVILA ÁLVAREZ, A.M. y DÍAZ MIER M.A. (2008). «Seis decenios de cooperación internacional: ¿qué hemos aprendido?». Revista ICE, n.. 843 .

[4] BARTON, J.; GOLDSTEIN, J.; JOSLYN, J. y STEINBENG, R. (2006). The evolution of the Trade regime. Princeton University Press.

[5] BERNHOFER, D.; FALVEY, R.; GREENAWAY, D. y KREICKERMEIR, U. (2011). Palgrave Handbook of International Trade. Palgrave MacMillan.

[6] CALVO HORNERO, M.A. (2016). Economía internacional y organismos económicos internacionales (2..$^{\text {a }}$ ed.). Ed. Ramón Areces.

[7] CATEGRA F.; GILLY, M. y GRAHAM, T. (2010). Marketing Internacional (3. - ed. en español). McGraw-Hill.

[8] COFFEY, F. y RILEY, R. (2006). Reforma of the International Institutions. Edward Elgar.

[9] DANIELS, J.; RADEBAUGH, L. y SULLIVAN, D. (2013). Negocios Internacionales: ambientes y operaciones (14. ${ }^{\mathrm{a}}$ ed.). Pearson.

[10] DUNNING, J.H. y LUNDAN, J.M. (2008). The Multinational Enterprises And the Global Economy (2..$^{\mathrm{a}}$ ed.). Edward Elgar.

[11] HEYDON, K. y WOOLCOCK (2012). The Ashgate Research Companion to International Trade Policy. Ed. Ashgate.

[12] HOEKMAN, B. y KOSTECKI, M. (2008). The Political Economy of the World Trade System (3. ${ }^{\text {a }}$ ed). Oxford University Press.

[13] JACKSON, JOHN H. (2006). Sovereignty, the WTO and Changing Fundamentals of International Law. Cambridge University Press.

[14] JACKSON, JOHN H. (2007). The Jurisprudence of GATT and the WTO. Cambridge University Press.

[15] MCGRORY, P.; APPLETON, A. y PLUMMER, M. (2005). The World Trade Organization: Legal, Economic and Political Analysis. Ed. Springer. 
[16] MATSUSHITA, M.; SCHOENBAUM, T.; MAVROIDIS, P. y HAHN, M. (2015). The World Trade Organization (3. ${ }^{\mathrm{a}}$ ed.). Oxford University Press.

[17] MOORE, M. (2005). «The Democratic Roots of the World Trade Organization», en la obra editada por McGrory citada (The World Trade Organization: Legal, Economic and Political Analysis, pp. 39-50).

[18] MORRISON, D. y AFILALO, A. (2008). The New Global Trading Order. Cambridge University Press.

[19] PETERSMAN, E.U. (2005). Reforming the World Trading System: Legitimacy, Efficient and Democratic Governance. Oxford University Press.

[20] SAMESON, G. (2001). The Role of the World Trade Organization in Global Governance. United Nations University Press.
[21] SITKING, A. y BOWEN, N. (2013). International Business: Challenges and Chains (8. ${ }^{a}$ ed.). Oxford University Press.

[22] SUTHERLAND, P. (2005). «The Politics of Trade Policy Development: The New Complexity", en la obra editada por MacGrory citada (The World Trade Organization: Legal, Economic and Political Analysis, pp. 27-38).

[23] SUTHERLAND, P. (2006). El futuro de la OMC: una respuesta a los desafíos Institucionales del nuevo milenio. Informe del Consejo Consultivo al Director General de la OMC, Ginebra.

[24] TREBILOCOCK, M.; HOWSE, R. y ELIASON, A. (2013). The Regulation of International Trade (4. ${ }^{\mathrm{a}}$ ed.). Routledge.

[25] WORTHINGTON, I. y BRISTON, C. (2015). The Business Environment (7. ${ }^{\text {a }}$ ed.). Pearson.

\section{ANEXO}

Temas que se tratan en el programa establecido en la Conferencia Ministerial de Doha con las mejoras realizadas en las Conferencias Ministeriales de Ginebra (2004) y Hong Kong (2005).

Agricultura

Algodón (introducido en 2004)

Servicios

Acceso a los mercados para productos no agrícolas (AMNA)

Equilibrio entre la agricultura y el AMNA (introducido en Hong Kong)

Propiedad intelectual

Subvenciones

Competencia

Transparencia de la contratación pública

Facilitación al comercio

Normas de la OMC. Antidumping

Normas de la OMC. Subvenciones

Normas de la OMC. Acuerdos regionales

Solución de diferencias

Medio ambiente

Comercio electrónico

Pequeñas economías

Comercio, deuda y finanzas
Comercio y transferencia de tecnología

Cooperación técnica

Países menos adelantados

Trato especial y diferenciado

Aplicación

ADPIC y salud pública

ADPIC y reclamaciones no basadas en una información (introducido en Hong Kong)

ADPIC y biodiversidad

ADPIC e inversiones geográficas

Marco integrado

Cuestiones relativas a los productos básicos (introducido en 2004)

Coherencia (introducido en 2005)

Ayuda para el comercio (introducido en Hong Kong)

Miembros de reciente adhesión (introducido en Hong Hong)

Adhesiones (introducido en Hong Kong)

Aplicación 